\title{
Emprendimiento corporativo y capital social: Un análisis de empresas intensivas en tecnología*
}

\author{
Delgado Márquez, Blanca L.** \\ Delgado Márquez, Luisa*** \\ Hurtado Torres, Nuria E.***
}

\section{Resumen}

El capital social constituye un activo estratégico de las empresas, facilitándoles el acceso y la explotación de nuevos recursos e influyendo positivamente en sus resultados. Dada la relevancia del capital social, resulta crucial conocer qué factores favorecen el desarrollo del mismo en el contexto organizacional. Por ello, en este trabajo se analiza la influencia del emprendimiento corporativo, variable que no solo contribuye a crear cambios económicos sino que también facilita la adaptación de las empresas a tales cambios, en el capital social de las empresas. El estudio empírico se centra en una muestra de 160 empresas de sectores intensivos en tecnología pertenecientes a países de la Unión Europea. La metodología consiste en la realización de análisis de regresión lineal jerárquica sobre dicha muestra. Los resultados obtenidos revelan la influencia positiva del emprendimiento corporativo sobre el desarrollo del capital social de las empresas, especialmente sobre su red de contactos, confianza, normas y reglas, concluyendo que el emprendimiento corporativo es clave en el desarrollo del capital social de las empresas.

Palabras clave: Emprendimiento corporativo, capital social, componentes del capital social, empresas de alta tecnología, Unión Europea.

Recibido: 10-02-12. Aceptado: 29-10-12

* Los autores desean expresar su más sincero agradecimiento a dos revisores anónimos por sus valiosas contribuciones en la mejora de este trabajo, así como al Ministerio de Educación español (proyecto ECO2010-20483) por la financiación otorgada.

** Profesora Ayudante Doctor. Departamento de Economía Internacional y de España. Facultad de Ciencias Económicas y Empresariales. Universidad de Granada, e-mail: bdelgado@ugr.es

*** Master en Economía y Organización de Empresas. Departamento de Organización de Empresas. Facultad de Ciencias Económicas y Empresariales. Universidad de Granada, e-mail: luisadm@correo.ugr.es

**** Profesora Titular de Universidad. Departamento de Organización de Empresas. Facultad de Ciencias Económicas y Empresariales. Universidad de Granada, e-mail: nhurtado@ugr.es 


\title{
Corporate Enterprise and Social Capital: An Analysis of Technology-Intensive Companies
}

\begin{abstract}
Social capital constitutes a strategic asset in companies, facilitating access to and exploitation of new resources and influencing their results in a positive manner. Given the relevance of social capital, it is crucial to know what factors favor its development in the organizational context. This study analyzes the influence of corporate enterprise, a variable that not only contributes to creating economic changes but also facilitates the adaptation of enterprises to such changes in the social capital of companies. This empirical study centers on a sample of 160 companies from technology-intensive sectors belonging to European Union countries. The methodology consists of performing a linear hierarchical regression analysis of the aforementioned sample. Results reveal the positive influence of corporate enterprise on social capital development in the companies, especially on their contact network, standards and rules, concluding that corporate enterprise is a key to developing the social capital of companies.
\end{abstract}

Key words: Corporate enterprise, social capital, social capital components, high technology enterprises, European Union.

\section{Introducción}

En el actual contexto económico, caracterizado por un elevado dinamismo y una alta complejidad, el capital social de las empresas desempeña un papel clave como activo estratégico (Govea y Rodríguez, 2009). En este sentido, el capital social permite a las empresas mejorar su posición de ventaja competitiva, y acceder y explotar de una forma eficiente una serie de recursos complementarios (Anandarajan et al., 2010; Mainardes et al., 2011). Asimismo, disponer de un adecuado capital social facilita el acceso de las empresas a la información y al conocimiento, una mejor coordinación de las actividades organizativas, el desarrollo de procesos de toma colectiva de decisiones y la reducción de los costes de transacción (Carpenter et al., 2012). El capital social contribuye positivamente al reconocimiento y aprovechamiento de nuevas oportunidades (Cegarra et al., 2011; Hotho y Champion, 2011; Shane y Venkataraman, 2000) y ejerce una influencia positiva sobre el desarrollo de actividades emprendedoras (Aldrich y Martínez 2003; Audretsch y Aldricht, 2012).

El capital social puede definirse como el conjunto de recursos arraigados dentro de una red de relaciones, así como todos los recursos accesibles a través de esa red (Nathapiet y Ghoshal, 1998). Las empresas pueden ser vistas como un conjunto de actores interdependientes conectados ${ }^{1}$ que transmiten conocimiento $y$ recursos (Simsek y Heavy, 2011). Por

1 Véase Gamboa et al. (2001) para una muy interesante discusión y propuesta de una tipología de las estrategias empresariales que refleja la conexión de la empresa con distintos actores, tales como otras empresas, clientes, gobierno, sindicato y la propia empresa. 
Emprendimiento corporativo y capital social: Un análisis de empresas intensivas... Delgado Márquez, Blanca L.; Delgado Márquez, Luisa y Hurtado Torres, Nuria E.

ello, tanto desde una perspectiva interpersonal como interorganizacional, el capital social hace referencia a la utilidad y beneficios derivados para los participantes de una red social (Carpenter, 2012), e incluye como constructo multidimensional, cinco componentes: confianza, creencias, normas, reglas y redes (McElroy et al., 2006).

Partiendo de la importancia del capital social para las empresas, estudios recientes han señalado la necesidad de desarrollar trabajos empíricos en los que se analice qué factores pueden influir en la generación de capital social (Hoang y Antonic, 2003; Payne et al., 2011). Por tal razón, el presente trabajo pretende responder a la llamada hecha por la literatura sobre la necesidad de profundizar empíricamente el análisis de los factores que permiten generar capital social, centrándose en cómo el emprendimiento corporativo influye en el desarrollo de capital social, trazándose como objetivo el análisis empírico de dicha relación, precisando la influencia que el emprendimiento corporativo tiene sobre cada uno de los componentes del capital social para el caso de empresas intensivas en tecnología. La metodología desarrollada se apoya en los análisis de regresión lineal jerárquica para evaluar el efecto de la variable independiente, el emprendimiento corporativo, sobre el capital social y sus componentes. Se selecciona una muestra de 160 empresas pertenecientes a sectores intensivos en tecnología, de una población total de 900 empresas de 10 países de la Unión Europea (Austria, Bélgica, Dinamarca, Francia, Alemania, Italia, Polonia, España, Holanda y Reino Unido). Se realizan encuestas vía telefónica a direc- tores de dichas empresas, apoyados en un instrumento de recolección de información construido en base a información documental previamente analizada. Una vez obtenido los resultados, éstos son analizados y contrastados con las hipótesis planteadas.

El emprendimiento corporativo implica adoptar una orientación a la innovación, una actitud proactiva y una mayor propensión a asumir riesgos (Lumpkin y Dess, 1996); es una capacidad dinámica que favorece ampliar y explotar recursos basados en el conocimiento que la empresa posee (Dess et al., 1997, Phan et al., 2009) y permite a las empresas anticiparse a los requerimientos de los mercados actuales, cada vez más dinámicos y competitivos. Además, el emprendimiento corporativo influye en el desarrollo de relaciones con otras empresas que permiten el intercambio de información valiosa, la toma de decisiones conjunta, y la coordinación y colaboración en el desarrollo de actividades (Simsek y Heavy, 2011).

En tal sentido, se pretende con este trabajo contribuir a enriquecer la literatura previa sobre capital social a través de dos importantes aportaciones; en primer lugar, se avanza en el estudio de los factores que influyen en el desarrollo de capital social, al analizar el mismo y sus componentes como variables dependientes, asumiendo el emprendimiento corporativo como variable generadora de capital social. Adicionalmente, el análisis de empresas intensivas en tecnología resulta especialmente relevante, puesto que los entornos a los que se enfrentan estas empresas son altamente competitivos y dinámicos, siendo la creación de capital social un activo especialmente estratégico y 
clave en la supervivencia y liderazgo de tales empresas. En segundo lugar, mediante el análisis desagregado del capital social en sus diferentes componentes, se ofrece una perspectiva más holística acerca de la influencia del emprendimiento sobre el capital social y, de esta forma, es posible alcanzar un mayor nivel de comprensión acerca de los procesos a través de los cuales las empresas generan capital social.

\section{Importancia del capital social en el contexto organizacional: Una aproximación teórica}

Son numerosas las definiciones que se han propuesto en la literatura sobre el capital social, así como las perspectivas bajo las cuales se ha analizado este concepto. No obstante, es posible identificar cuatro enfoques principales en la conceptualización del término: comunitario, de red, institucional y sinérgico (Woolcock y Narayan, 2000).

Siguiendo el enfoque comunitario, algunos autores han equiparado el capital social con organizaciones locales, como clubes, asociaciones o grupo cívicos. Desde este punto de vista, sostienen que el capital social es inherentemente beneficioso y tiene un impacto positivo en el bienestar de una comunidad (Fukuyama, 1995, 1997; Putnam, 1993, 1995). Por su parte, el enfoque de red argumenta que el capital social potencial de un individuo u organización consiste en el conjunto de relaciones en las que dicho individuo u organización está involucrado y a las cuales tiene acceso, así como la forma en la que este conjunto de relaciones está estructurado. Desde esta perspectiva, se destaca la importancia de las relaciones entre los individuos, y entre las organizaciones (Bourdieu, 1986; Burt, 1992, 1997, 2001; Coleman, 1988, 1990; Portes, 1998). El enfoque institucional percibe el capital social como una variable dependiente del entorno político, jurídico e institucional. A diferencia de los enfoques comunitarios y de red, que tradicionalmente han identificado el capital social como una variable independiente, el enfoque institucional lo considera como variable dependiente. Asimismo, este enfoque pone especial énfasis en el análisis de políticas macroeconómicas, dejando de lado el aspecto microeconómico (Woolcock y Narayan, 2000). Finalmente, el enfoque sinérgico trata de integrar los enfoques de red e institucional (Narayan, 1999; Woolcock, 1998). Este enfoque identifica el capital social con la naturaleza y el grado de las relaciones sociales de una comunidad y sus instituciones formales, así como con la interacción que tiene lugar entre ellos.

En este trabajo se adopta el enfoque de red, asumiendo la definición de capital social propuesta por Nahapiet y Ghoshal (1998), quienes lo definen como el conjunto de recursos actuales y potenciales arraigados y derivados de la red de relaciones que posee un individuo o unidad social, así como todos los recursos accesibles a través de esa misma red.

Tanto desde una perspectiva interpersonal como interorganizacional, el capital social hace referencia a la utilidad y beneficios derivados de una red social para sus participantes (Burt, 1997; Carpenter et al., 2012), potencia, entre otros aspectos, un mayor nivel de innovación 
Emprendimiento corporativo y capital social: Un análisis de empresas intensivas... Delgado Márquez, Blanca L.; Delgado Márquez, Luisa y Hurtado Torres, Nuria E.

en las empresas (Shipilov, 2009), un mejor acceso a los recursos (Shane y Cable, 2002), unos menores costos de transacción (Gulati, 1995) y contribuye a un mejor rendimiento organizacional (Peng y Luo, 2000).

A través de las relaciones interorganizativas (capital social) se generan oportunidades para la adquisición y explotación de conocimiento externo (Lane y Lubatkin, 1998; Simsek y Heavy, 2011), derivado del capital social organizativo, que puede favorecer el desarrollo de innovaciones en productos y procesos y ayudar a las empresas a expandirse a nuevos mercados (Thorgren et al., 2009).

Los beneficios del capital social en términos de generación de conocimiento resultan especialmente relevantes en el contexto de sectores intensivos en tecnología, caracterizados por rasgos de elevada competitividad e innovación que favorecen el desarrollo de nuevos productos, la distinción tecnológica y la eficiencia en costos (Yli-Renko et al., 2001). La generación y explotación de conocimiento en sectores intensivos en tecnología requiere que el conocimiento sea continuamente renovado (Lane y Lubatkin, 1998), pues su creación y explotación son procesos eminentemente sociales (Kogut y Zander, 1992), siendo el capital social crítico para la supervivencia de las empresas intensivas en tecnología.

Este hecho resulta especialmente crucial en el actual contexto económico, en el que la economía mundial ha evolucionado hacia un sistema basado en el conocimiento y se encuentra caracterizada por mercados y tecnologías rápidamente cambiantes (Doh y Zolnik, 2011). Por todo ello, es relevante profundizar en el conocimiento de los factores que favorecen el desarrollo del capital social.

\section{El emprendimiento corporativo y el capital social}

En el área del capital social, uno de los conceptos que ha recibido una mayor atención tanto desde el punto de vista teórico como empírico ha sido el emprendimiento corporativo (Rauch et al., 2009). En el Cuadro 1 se resume una perspectiva comparada de algunos de los principales trabajos.

Según Rodríguez et al. (2010), diferentes perspectivas teóricas justifican el origen del emprendimiento, la perspectiva comportamental hace referencia a la influencia que la personalidad del individuo puede tener en el desarrollo de la actividad emprendedora; la perspectiva psicológica o cognitiva, por su parte, identifica el emprendimiento corporativo como una característica inherente al individuo; la perspectiva de procesos sitúa al individuo como centro del argumento, en una relación biyectiva entre su propio desarrollo y la creación de valor económico; la perspectiva cultural sostiene que tanto la mentalidad del propio emprendedor como las influencias que recibe de agentes externos afectan al tipo de acciones que éste emprende para aprovechar oportunidades existentes en el entorno. Finalmente, la perspectiva psicoanalítica bucea en los rasgos adquiridos por el individuo desde el momento de su nacimiento y que le confieren una forma de percibir la realidad que determina el modo de comportarse a la hora de desarrollar o no un comportamiento basado en el emprendimiento corporativo. 


\section{Cuadro 1 \\ Influencia del emprendimiento corporativo sobre el capital social: Hallazgos previos}

\begin{tabular}{cl}
\hline \multicolumn{1}{c}{ Autores } & Influencia del emprendimiento sobre el capital social \\
\hline Simsek y Heavy (2011) & $\begin{array}{l}\text { El emprendimiento corporativo favorecerá el mantener relaciones con } \\
\text { otras empresas y construir otras nuevas que permitan el intercambio } \\
\text { de información sustanciosa, coordinación, colaboración y toma de de- } \\
\text { cisiones conjuntas entre las distintas entidades. }\end{array}$ \\
Hayton y Kelley (2006) & $\begin{array}{l}\text { El emprendimiento corporativo, al necesitar acceder e integrar dife- } \\
\text { rentes fuentes de conocimiento a través de la organización, fomenta } \\
\text { los vínculos sociales entre individuos y unidades. }\end{array}$ \\
Morris et al. (2008) & $\begin{array}{l}\text { El emprendimiento corporativo facilita las interacciones entre directi- } \\
\text { vos de alto y medio nivel para el aprovisionamiento de recursos nece- } \\
\text { sarios para el desarrollo y legitimación de iniciativas emprendedoras. } \\
\text { Kuratko et al. (2005) }\end{array}$ \\
$\begin{array}{l}\text { El emprendimiento corporativo favorece el desarrollo de interacciones } \\
\text { entre directivos de medio y bajo nivel de la empresa para la planifica- } \\
\text { ción y organización de las iniciativas emprendedoras. }\end{array}$ \\
\hline
\end{tabular}

Fuente: Elaboración propia.

En tal sentido, se define el emprendimiento corporativo como una capacidad dinámica que permite explotar y ampliar los recursos basados en el conocimiento que posea la empresa (Phan et al., 2009; Zahra et al., 1999). Implica adoptar una actitud proactiva, una orientación a la innovación y una propensión a asumir riesgos en todas las actividades desarrolladas en la empresa (Lumpkin y Dess, 1996) ${ }^{2}$. En cuanto a los elementos anteriores, la proactividad puede ser definida como la capacidad de anticiparse a los mercados, aprovechando las oportunidades de negocio emergentes y creando una ventaja con respecto a sus competidores; la orientación a la innovación, hace referencia a la habilidad de la em- presa para desarrollar nuevos productos, modificar los ya existentes y fomentar la experimentación, refleja la tendencia a apoyar nuevas ideas, lo novedoso y la experimentación en procesos creativos (Lumpkin y Dess, 1996); por último, la propensión a asumir riesgos implica una disposición más favorable a tomar decisiones que comprometan recursos, sin certeza sobre las posibles ganancias y en las que los costos derivados del fracaso pueden ser altos.

La importancia del emprendimiento corporativo radica en el hecho de que favorece la acumulación y el apalancamiento de recursos que permiten alcanzar los objetivos competitivos de la empresa, tales como el desarrollo de nuevos

2 Rodríguez et al. (2010) plantea en su artículo una muy interesante discusión acerca de la evolución del concepto de emprendimiento. 
Emprendimiento corporativo y capital social: Un análisis de empresas intensivas... Delgado Márquez, Blanca L.; Delgado Márquez, Luisa y Hurtado Torres, Nuria E.

productos o procesos, la introducción de innovaciones administrativas o la entrada en nuevos mercados (Covin y Miles, 1999). En este sentido, Zahra et al. (1999:169) manifiestan que "las actividades formales e informales del emprendedor corporativo pueden mejorar el resultado organizacional de la empresa a través de la creación de nuevo conocimiento que se convierta en la base para el desarrollo de nuevas competencias o la revitalización de las ya existentes".

Siguiendo esta misma línea, Teng (2007:122) señala que "una visión global del emprendimiento corporativo consiste en la búsqueda de oportunidades que permitan ampliar la base de recursos de la empresa sin romper del todo con su base de recursos ya existente", lo que implica el desarrollo de nuevo conocimiento a partir de viejas rutinas, sistemas y procesos por los que se regía antes la empresa (Hayton y Kelley, 2006; Zahra et al., 1999).

El emprendimiento corporativo facilita las interacciones entre los directivos de alto y medio nivel tanto para planificar y organizar iniciativas estratégicas como para conseguir el aprovisionamiento de los recursos necesarios para llevar a cabo iniciativas emprendedoras así como su legitimación (Kuratko et al., 2005 y Morris et al., 2008). Necesitará acceder e integrar diferentes fuentes de conocimiento a través de la organización (Hayton y Kelley, 2006) y, con tal objetivo, fomentar los vínculos sociales entre diferentes individuos y unidades, es decir, apoyar el capital social interno de la empresa (Simsek y Heavy, 2011).

Por otra parte, el emprendimiento corporativo de una empresa también favorece el mantenimiento de sus relaciones con otras empresas, asi como la construcción de otras nuevas que permitan el intercambio de información sustanciosa, coordinación y colaboración, así como de toma de decisiones conjuntas entre diversas empresas (Simsek y Heavy, 2011). Los mayores niveles de emprendimiento permiten identificar y valorar ideas y oportunidades que otras empresas han pasado por alto y no han explotado y reconocer el valor de los recursos y capacidades (Álvarez y Busenitz, 2001). Por su parte, Crant (2000) reconoce que la falta de proactividad obstaculiza a las empresas el identificar y aprovechar oportunidades. En base a lo expuesto, cabe esperar que si una empresa posee mayores niveles de emprendimiento corporativo, será capaz de reconocer el valor que se puede derivar del capital social, lo que influirá positivamente en el desarrollo del mismo. Tomando como base esta afirmación, se formula la siguiente hipótesis:

Hipótesis 1: El emprendimiento corporativo influye positivamente en el capital social.

El capital social, como constructo multidimensional, puede ser analizado a través de una serie de componentes: confianza, creencias, normas, reglas y redes (McElroy et al., 2006; Smedlund, 2008).

La confianza se define como un estado psicológico que implica la intención de situarse en una posición de vulnerabilidad a las acciones de otro basándose en expectativas positivas acerca de las intenciones o comportamientos del otro (Rousseau et al., 1998), la confianza "favorece la cooperación y la cooperación en sí, engendra confianza" (Putnam, 1993: 171). La literatura sobre confianza propone que las decisiones tomadas por 
aquellos agentes que van a decidir si confiar o no implican un nivel de riesgo que depende de otros agentes con los que la decisión está relacionada (esto, es, aquellos agentes en los que se va a confiar). La confianza implica, pues, incertidumbre y la asunción de cierto nivel de riesgo (Delgado et al., 2012), este último definido como la probabilidad de pérdida desde la óptica del agente que decide si confiar (Coleman, 1990; Williamson, 1993). Dado que el emprendimiento corporativo implica, entre otros, la asunción de riesgos en la toma de decisiones que puedan comprometer ingentes cantidades de recursos de la empresa, cabe esperar que aquellas organizaciones con un mayor emprendimiento corporativo tengan una mayor propensión a asumir los riesgos inherentes a las decisiones de confianza involucradas en el desarrollo de un mayor capital social. Por ello, se plantea la siguiente hipótesis:

Hipótesis 1a: El emprendimiento corporativo influye positivamente en la confianza de las empresas en sus relaciones con otras empresas.

Las creencias constituyen otro componente del capital social. Tal y como afirma Coleman (1990), las creencias compartidas son un aspecto fundamental para llevar a cabo la acción colectiva, dado que aseguran que los individuos están persiguiendo los mismos objetivos (Smedlund, 2008) y guían la estrategia organizacional (Romero, 2006). A nivel interorganizativo, si no existen buenas relaciones entre las empresas basadas en el compromiso y una visión o creencias similares, las empresas son reacias a compartir información (Li y Lin, 2006). Es importante tener en cuenta que la creación de unos esque- mas o creencias comunes es un proceso que implica tiempo para compartir experiencias, interacciones y la resolución conjunta de problemas (Schein, 1985). Así pues, cabe esperar que las organizaciones con un mayor emprendimiento corporativo, dada su mayor proactividad, orientación a la innovación y propensión a asumir riesgos, fomenten el desarrollo de unos esquemas de creencias compartidos tanto entre sus miembros, como con aquellas empresas con las que tienen establecidos vínculos o con las que desean entablarlos. Por tanto, se plantean las siguientes hipótesis:

Hipótesis 1b: El emprendimiento corporativo influye positivamente en las creencias compartidas entre las empresas de una red.

Por otra parte, las normas, al ser el resultado de un conjunto de acciones que son consideradas por individuos como correctas o incorrectas, únicamente pueden alcanzarse cuando hay una comunicación regular entre los individuos (Coleman, 1990). Las normas que permiten desarrollar capital social son aquellas que fomentan la cooperación y, por tanto, están relacionadas con valores tradicionales como la honestidad, el mantenimiento de los compromisos adquiridos o la reciprocidad (Fukuyama, 2001). De este modo, las normas constituyen medios para alcanzar fines cooperativos. Estudios previos han demostrado que la coordinación basada en normas, considerado un mecanismo de control informal, desempeña un papel crucial en las economías modernas, e incluso alcanza una mayor relevancia a medida que la naturaleza de la actividad económica se hace más compleja y tecnológicamente 
Emprendimiento corporativo y capital social: Un análisis de empresas intensivas... Delgado Márquez, Blanca L.; Delgado Márquez, Luisa y Hurtado Torres, Nuria E.

sofisticada (Fukuyama, 1999). Dentro de los sectores intensivos en tecnología, estudios previos han encontrado que la investigación y desarrollo en alta tecnología a menudo depende del intercambio informal de derechos de propiedad intelectual, sencillamente porque los mecanismos de intercambio formal implicarían unos costos de transacción muy elevados que ralentizarian la velocidad de intercambio (Saxenian, 1994). En este sentido, cabe esperar que las organizaciones con un mayor emprendimiento corporativo, fomenten el desarrollo de mecanismos de control informal o de normas que favorezca la cooperación con otras empresas, aspecto clave para actuar en el entorno competitivo más complejo y dinámico. En base a ello, se plantea la siguiente hipótesis:

Hipótesis 1c: El emprendimiento corporativo influye positivamente en el desarrollo de normas que fomentan la cooperación.

Otro componente del capital social son las reglas consideradas mecanismos de control formales que requieren llevar a cabo inversiones en autoridad y sanciones que permitan alcanzar un funcionamiento efectivo de la organización (Coleman, 1990). Tanto las reglas como las normas son afirmaciones que pueden permitir, obligar o prohibir una acción, no obstante, mientras una regla incluye una o más especificaciones o sanciones que la refuerzan, las normas no (Ostrom, 2005). Así, cuando los mecanismos sancionadores establecidos por la empresa hacen que la ruptura de los acuerdos sea costosa para el agente que viola lo establecido, los agentes estarán más dispuestos a cooperar entre sí (Doh y Zolnik,
2011). La implementación de reglas favorece el intercambio de información confidencial entre empresas, puesto que disminuye el riesgo de que una organización se comporte de forma oportunista y explote de forma inadecuada dicha información confidencial, perjudicando a las otras empresas (Dakhli y de Clercq, 2004). En este sentido, en aquellos contextos en los que los mecanismos informales o normas no sean efectivos, la implementación de reglas que fomenten la cooperación y el intercambio de información y conocimiento es deseable para favorecer los procesos de innovación y creación. La acumulación de conocimiento permite a las organizaciones comprender y evaluar mejor el potencial comercial de sus innovaciones y avances tecnológicos. Por ello, aquellas organizaciones con un mayor emprendimiento corporativo serán más proclives a desarrollar reglas que eviten comportamientos oportunistas que comprometan la transferencia de conocimiento e información y, por tanto, favorecerán el desarrollo de capital social. En base a lo anteriormente comentado, se plantea la siguiente hipótesis:

Hipótesis 1d: El emprendimiento corporativo influye positivamente en el desarrollo de reglas que eviten comportamientos oportunistas.

Finalmente, las redes constituyen otro componente del capital social, estas se definen como las conexiones existentes entre dos o más personas u organizaciones (Smedlund, 2008). Estudios recientes subrayan que el desarrollo de redes puede mejorar la eficiencia de la sociedad facilitando el desarrollo de acciones coordinadas (Fernández et al., 2010) y el aprendizaje intraorganizativo (Sánche et al., 2010). A 
través de las redes, las organizaciones tienen acceso a información y conocimiento de utilidad y pueden tomar decisiones basadas en estos acerca de un conjunto de alternativas disponibles (Doh y Zolnik, 2011). Asimismo, las redes crean nuevas oportunidades a través de la internalización de las habilidades de otros agentes pertenecientes a la misma red. Por ejemplo, si las empresas de alta tecnología están integradas en una red, su participación es señal de riesgo reducido para los inversores (Podolny, 2001). De este modo, cabe esperar que las organizaciones con un mayor emprendimiento corporativo, que se caracterizan por niveles más elevados de proactividad y, por tanto, son conscientes de las numerosas oportunidades de negocio que pueden derivarse de los contactos a través de estas redes, fomenten el desarrollo y configuración de redes. En base a lo comentado anteriormente, se establece la siguiente hipótesis:

Hipótesis 1e: El emprendimiento corporativo influye positivamente en el desarrollo de redes.

Dentro del contexto del emprendimiento corporativo, estos componentes del capital social presentan una conceptualización que se resume en el Cuadro 2.

\section{Influencia del emprendimiento corporativo sobre el capital social: Un análisis empírico}

Para la contrastación de las mencionadas hipótesis, el estudio se centró en empresas pertenecientes a sectores intensivos en tecnología, debido al interés inherente que conlleva el estudio del capital social y el emprendimiento corporativo en aquellos sectores con un alto nivel tecnológico. En la obtención de los datos se ha seguido una aproximación sistemática. En primer lugar, tomando como base literatura previa (Frohlich y Westbrook, 2002), se diseñó un cuestionario con el que se realizó un estudio piloto en el que participaron 8 directivos de empresas de los sectores considerados.

La población total estaba constituida por 900 empresas pertenecientes a 10 países de la Unión Europea (Austria, Bélgica, Dinamarca, Francia, Alemania, Italia, Polonia, España, Holanda y Reino Unido). La selección de la muestra se realizó a partir de la base de datos Amadeus, empleando para ello un muestreo estratificado de asignación óptima. De acuerdo con esta técnica y, dado que en todos los países las empresas que formaban la población presentaban una variabilidad muy similar entre sí, una submuestra representativa de las empresas de cada país, o en su defecto de cada estrato, debe de estar constituida por 16 empresas seleccionadas de forma aleatoria en cada uno de los estratos (Luque, 2000), se obtuvo como resultado una muestra final de 160 empresas.

Una vez seleccionada la muestra, se procedió a la elaboración de un listado de los directores generales de estas empresas. Las razones que justifican el hecho de escoger a los directores generales como informantes, fueron varias. 1) poseen un gran conocimiento respecto a todos los departamentos de su compañía; 2) constituyen una valiosa fuente de evaluación y modulación de las distintas variables de estudio, determinando la conducta que es de esperar y apoyar (Baer y 
Emprendimiento corporativo y capital social: Un análisis de empresas intensivas... Delgado Márquez, Blanca L.; Delgado Márquez, Luisa y Hurtado Torres, Nuria E.

\section{Cuadro 2 \\ Componentes del capital social en el contexto del emprendimiento corporativo}

\begin{tabular}{ll}
\hline Componente & \\
\hline Confianza & La confianza implica asumir riesgos. Aquellas empresas que exhiban un ma- \\
& yor emprendimiento corporativo tendrán, una tendencia a asumir los riesgos \\
& inherentes a las relaciones de confianza y las promoverán como componente \\
& del capital social. \\
Creencias & Las creencias compartidas desempeñan un papel fundamental en la acción \\
& colectiva que caracteriza los contextos organizacionales. Las empresas con \\
& mayor emprendimiento corporativo promoverán el desarrollo de unos esque- \\
& mas de creencias compartidos entre sus miembros así como con aquellas em- \\
& presas con las que tienen establecidos vínculos o con las que desean enta- \\
& blarlos. \\
Normas & Las normas, como mecanismos de control informal, favorecen el comporta- \\
& miento cooperativo en el contexto organizacional evitando retardos. Las em- \\
& presas con mayor emprendimiento corporativo, dada su mayor orientación \\
& proactiva, promoverán mecanismos informales. \\
& Las reglas previenen los comportamientos oportunistas que destruyen el capi- \\
tal social en el contexto organizacional. Por ello, las empresas con un mayor & emprendimiento corporativo favorecerán el desarrollo de reglas en aquellas \\
Reglas & situaciones en las que los mecanismos de control informal no sean factibles. \\
& Las redes, como conjunto de conexiones entre organizaciones, constituyen \\
una importante fuente de acceso a información y conocimiento. Las organiza- \\
ciones con un mayor emprendimiento corporativo, conscientes de las oportu- \\
nidades de negocio que pueden derivarse de tales conexiones, favorecerán el \\
desarrollo de estas redes.
\end{tabular}

Fuente: Elaboración propia.

Frese, 2003); 3) son los responsables, en última instancia, de planear la organización y objetivos de la empresa así como las medidas que se han de llevar a cabo para poder alcanzar tales fines (Westhpal y Fredickson, 2001).

El procedimiento seguido para la obtención de los datos de la muestra fue la encuesta telefónica. Con el objetivo de reducir la existencia de sesgo de no respuesta, se compararon las características de los negocios de aquellas empresas que no habían respondido respecto a las que sí lo habían hecho. Los resultados obtenidos respecto a la rentabilidad económica, el patrimonio y el porcentaje de beneficios sobre el total de facturación así como el número de empleados indicó que no existían diferencias significativas entre las empresas que habían respondido y las que no (Armstrong y Overton, 1977). Dado que todas las medidas del análisis de investigación estaban incluidas en el mismo cuestionario, se probó el método común del sesgo por medio del test de un solo factor de Harman (Konrad y Linnehan, 1995). El Cuadro 3 recoge información detallada acerca de la compo- 


\section{Cuadro 3}

Características de la muestra

\begin{tabular}{ll}
\hline Sectores & $\begin{array}{l}\text { Industria farmacéutica, hardware y otros equi- } \\
\text { pos informáticos, industria automovilística, pro- } \\
\text { ductos espaciales y aeronáuticos. }\end{array}$ \\
$\begin{array}{l}\text { Países y número de entrevistas a directivos } \\
\text { y académicos en cada país }\end{array}$ & $\begin{array}{l}\text { cus (16), Bélgica (16), Dinamarca (16), Fran- } \\
\text { España (16), Holanda (16) y Reino Unido (16), }\end{array}$ \\
Tamaño de la muestra & 160 empresas \\
Metodología & Encuesta telefónica \\
Ratio de respuesta & $17.7 \%$ \\
Periodo de recolección de datos & Desde mayo de 2010 a septiembre de 2010 \\
\hline
\end{tabular}

Fuente: Elaboración propia.

sición de la muestra y el procedimiento empleado para seleccionarla.

Para medir el capital social se empleó una escala Likert de 1 a 7 puntos. Esta escala está basada en la propuesta por Kale et al. (2000) y Knight (1997), formada por 10 ítems que recogen la medición de las diferentes componentes del capital social: red, confianza, creencias, normas y reglas. La variable final, capital social, se obtuvo como media de estos ítems. Para determinar la fiabilidad de medida de los ítems seleccionados, se procedió al cálculo del alfa de Cronbach, obteniendo un valor 0.727 , puntuación que demuestra una perfecta adecuación de los ítems al concepto de capital social.

La medición de cada componente del capital social se realizó en base a la misma escala, seleccionando los ítems correspondientes a la medición de cada componente. La variable final en cada caso se calculó como la media de estos ítems. En todos los casos, los valores del indicador alfa de Cronbach revelan la validez de las escalas empleadas: 0.692 (componente red), 0.701 (componente confianza), 0.483 (componente creencias), 0.623 (componente normas) y 0.538 (componente reglas).

La medición del emprendimiento a nivel corporativo se sustentó en una escala basada en la propuesta por Covin y Slevin (1988, 1989), y que contiene 9 ítems que miden la tendencia a innovar de la empresa, su capacidad para asumir riesgos y su proactividad. Todos estos aspectos han sido descritos como las subdimensiones del concepto emprendimiento corporativo (Miller, 1983) y contrastadas por numerosos trabajos empíricos. Para asegurar la fiabilidad y validez de la escala de medida se calculó el alfa de Cronbach, obteniendo un valor 0.807 , dato que revela la adecuación de los ítems seleccionados para medir el concepto de emprendimiento corporativo. La variable final, emprendimiento corporativo, se obtuvo como media de estos ítems.

Teniendo en cuenta los efectos potenciales del tamaño sobre el capital social y sus componentes, se incluyó el tamaño como variable control. El tamaño 
Emprendimiento corporativo y capital social: Un análisis de empresas intensivas... Delgado Márquez, Blanca L.; Delgado Márquez, Luisa y Hurtado Torres, Nuria E.

se midió a través del número de empleados (Covin y Slevin, 1989), utilizando en el modelo una transformación logarítmica con el objetivo de reducir la varianza de la distribución de valores del tamaño a lo largo de la muestra (Kimberly y Evanisko, 1981). Adicionalmente, se incluyeron como variables control el país y el sector de actividad al que pertenecía la empresa (Covin y Slevin, 1989).

Dada la distribución continua seguida por las variables dependientes, el capital social y sus componentes, se emplearon análisis de regresión jerárquica (Cohen y Cohen, 1983) para evaluar el efecto de la variable independiente, el emprendimiento corporativo, sobre el capital social y sus componentes. Las inter-correlaciones para todas las variables explicativas -el emprendimiento corporativo y las variables control- se han examinado empleando tanto correlaciones bivariadas como factores de inflación de las varianzas (FIV). El primer método muestra que las inter-correlaciones para todas las variables explicativas son inferiores a 0.7 (véanse los estadísticos descriptivos y la matriz de correlaciones para el capital social y sus compo- nentes en las Tablas 1 y 2, respectivamente). Por su parte, el análisis FIV revela que no existen signos de multicolinealidad, y los valores FIV de todas las variables explicativas varian entre $1.008 \mathrm{y}$ 1.082 , esto es, son considerablemente inferiores al "límite máximo superior de 10 considerado como aceptable" (Hair et al., 2006: 230). Así pues, ambos tests sugieren que los estimadores de regresión no se ven degradados por la presencia de multicolinealidad. La Tabla 1 muestra los elevados niveles medios tanto del emprendimiento corporativo como del capital social agregado de las empresas de alta tecnología analizadas. Asimismo, la Tabla 2 evidencia que, si bien los valores medios de todos los componentes del capital social son elevados, las reglas, la confianza y las redes tienen una mayor presencia en las empresas objeto de estudio. Por el contrario, el valor medio de las creencias es inferior a la media de los demás componentes del capital social.

Los resultados de las regresiones jerárquicas para el capital social y sus componentes se recogen en las Tablas 3 y 4 , respectivamente. Ambas tablas muestran los coeficientes estandariza-

Tabla 1

Estadísticos descriptivos y matriz de correlaciones para el capital social

\begin{tabular}{lcccccc}
\hline \multicolumn{1}{c}{ Variable } & Media & $\begin{array}{c}\text { Desviación } \\
\text { típica }\end{array}$ & $\mathbf{1}$ & $\mathbf{2}$ & $\mathbf{3}$ & $\mathbf{4}$ \\
\hline 1. Emprendimiento corporativo & 4.81 & 1.21 & & & & \\
2. País & - & - & $.138^{*}$ & & & \\
3. Tamaño de la empresa & - & - & $.208^{* *}$ & $.197^{* *}$ & & \\
4. Sector de actividad & - & - & -.033 & $-.201^{* *}$ & $-.127+$ & \\
5. Capital social & 5.03 & 1.06 & $.289^{* * *}$ & .078 & $.157^{*}$ & .016 \\
\hline
\end{tabular}

${ }^{+} p<.10^{*} p<.05^{* *} p<.01{ }^{* * *} p<.001$.

Fuente: Elaboración propia. 


\section{Tabla 2}

\section{Estadísticos descriptivos y matriz de correlaciones para los componentes del capital social}

\begin{tabular}{|c|c|c|c|c|c|c|}
\hline Variable & Media & $\begin{array}{c}\text { Desviación } \\
\text { típica }\end{array}$ & 1 & 2 & 3 & 4 \\
\hline $\begin{array}{l}\text { 1. Emprendimiento } \\
\text { corporativo }\end{array}$ & 4.81 & 1.21 & & & & \\
\hline 2. País & & & $.138^{*}$ & & & \\
\hline 3. Tamaño de la empresa & & & $.208^{* *}$ & $.197^{* *}$ & & \\
\hline 4. Sector de actividad & & & -.033 & $-.201^{* *}$ & $-.127+$ & \\
\hline 5. Componente red & 5.18 & 1.730 & $.221^{* *}$ & .096 & $.130+$ & .014 \\
\hline 6. Componente confianza & 5.24 & 1.689 & $.228^{\star *}$ & .069 & $.178^{*}$ & .047 \\
\hline 7. Componente creencias & 4.58 & 1.789 & .073 & -.052 & $.103^{+}$ & .023 \\
\hline 8. Componente normas & 4.96 & 1.417 & $.154^{*}$ & .050 & -.063 & .007 \\
\hline 9. Componente reglas & 5.32 & 1.142 & $.449^{* \star \star}$ & $.175^{\star}$ & $.136^{*}$ & -.029 \\
\hline
\end{tabular}

Tabla 3

Resultados del análisis con regresión jerárquica (variable dependiente: Capital social).

\begin{tabular}{lcc}
\hline \multicolumn{1}{c}{ Variables } & Modelo 1 & Modelo 2 \\
\hline País & .103 & .088 \\
Tamaño de la empresa & $.189^{*}$ & $.150^{+*}$ \\
Sector de actividad & .011 & -.001 \\
Emprendimiento corporativo & & $.258^{* *}$ \\
$\mathrm{R}$ & .230 & .343 \\
$\mathrm{R}^{2}$ & .053 & .118 \\
Cambio en $\mathrm{R}^{2}$ & $.053^{*}$ & $.065^{\star *}$ \\
$\mathrm{R}^{2}$ ajustada & .035 & .095 \\
\hline Todos los coeficientes representan valores estandarizados. ${ }^{+} p<.10^{*} p<.05^{* *} p<.01^{* * *} p<.001$ \\
Fuente: Elaboración propia.
\end{tabular}

dos que indican el efecto que un cambio en una unidad estándar en una variable independiente tiene sobre la variable dependiente. La Tabla 3 recoge la información referente al capital social en general. El modelo 1 constituye el modelo base e incluye exclusivamente las variables con- trol, que conjuntamente explican el 3.5\% de la variabilidad de los datos $\left(R^{2}\right.$ ajustada). De las tres variables control analizadas, solamente el tamaño de la empresa ejerce una influencia significativa en el nivel de capital social. En el modelo 2, construido para testar la hipótesis 1 , se 
Emprendimiento corporativo y capital social: Un análisis de empresas intensivas... Delgado Márquez, Blanca L.; Delgado Márquez, Luisa y Hurtado Torres, Nuria E.

investiga una potencial influencia adicional en el nivel de capital social. Este modelo evidencia la existencia de una influencia positiva del grado de emprendimiento corporativo sobre el nivel de capital social general de las empresas, hecho que confirma la hipótesis 1 . Asimismo, el tamaño de la empresa continúa siendo determinante y la introducción del emprendimiento corporativo como variable independiente contribuye a mejorar la explicación de la variabilidad de los datos hasta situarla en un $9,5 \%$.

La Tabla 4 resume la información sobre las regresiones jerárquicas referidas a cada una de los componentes del capital social. Para cada componente se han testado dos modelos: un modelo base que investiga la influencia de las variables control (Modelo 1), y un modelo que sirve para analizar la influencia del emprendimiento corporativo en esa componente del capital social (Modelo 2). De todos estos análisis cabe extraer varias conclusiones. Por un lado, respecto a los modelos base, ninguna variable control parece ejercer una influencia estadísticamente significativa sobre ningún componente del capital social, salvo en el caso del tamaño de la empresa sobre el componente confianza y de la variable país en el componente reglas. Por otro lado, en relación a los cinco modelos que sí incorporan el emprendimiento corporativo, los datos muestran que la inclusión del emprendimiento corporativo como variable independiente mejora la variabilidad de los datos en comparación con los modelos de partida. Concretamente, el emprendimiento corporativo ejerce una influencia positiva y estadísticamente significativa sobre todas los componentes del capital social, a excepción del componente creencias. Es- tos resultados apoyan las hipótesis $1 \mathrm{a}$, $1 \mathrm{c}, 1 \mathrm{~d}$ y $1 \mathrm{e}$, refutando la hipótesis $1 \mathrm{~b}$.

Así pues, los resultados ofrecen apoyo a la mayoría de las hipótesis planteadas. En primer lugar, en relación a la hipótesis 1 , los resultados apoyan que el emprendimiento corporativo constituye un activo estratégico de las empresas a la hora de generar capital social corporativo. Ello es debido a que la actitud más proactiva vinculada a las organizaciones más emprendedoras les permite reconocer el valor de acceder y explotar nuevos recursos y contactos, derivados del desarrollo del capital social corporativo. En segundo lugar, la evidencia empírica sustenta la hipótesis $1 \mathrm{a}$, de acuerdo a la cual el emprendimiento corporativo, al implicar una actitud empresarial más propensa a asumir los riesgos, ejerce una influencia positiva en la confianza de una empresa en sus relaciones con otras empresas. En tercer lugar, los datos revocan la influencia del emprendimiento corporativo sobre las creencias, planteada en la hipótesis $1 \mathrm{~b}$. Esto puede ser debido a que el desarrollo de esquemas compartidos de creencias requiere participar conjuntamente en experiencias, así como en la resolución de problemas. Por ello, la no influencia del emprendimiento corporativo sobre el componente creencias puede estar ocasionada por el carácter transversal del análisis empírico realizado. En cuarto lugar, los análisis realizados apoyan la hipótesis 1c, que establece que las organizaciones más emprendedoras, al ser plenamente conscientes del valor que los mecanismos de control informal como las normas tienen para desarrollar con éxito la actividad empresarial en entornos complejos y dinámicos, fomentan estos 


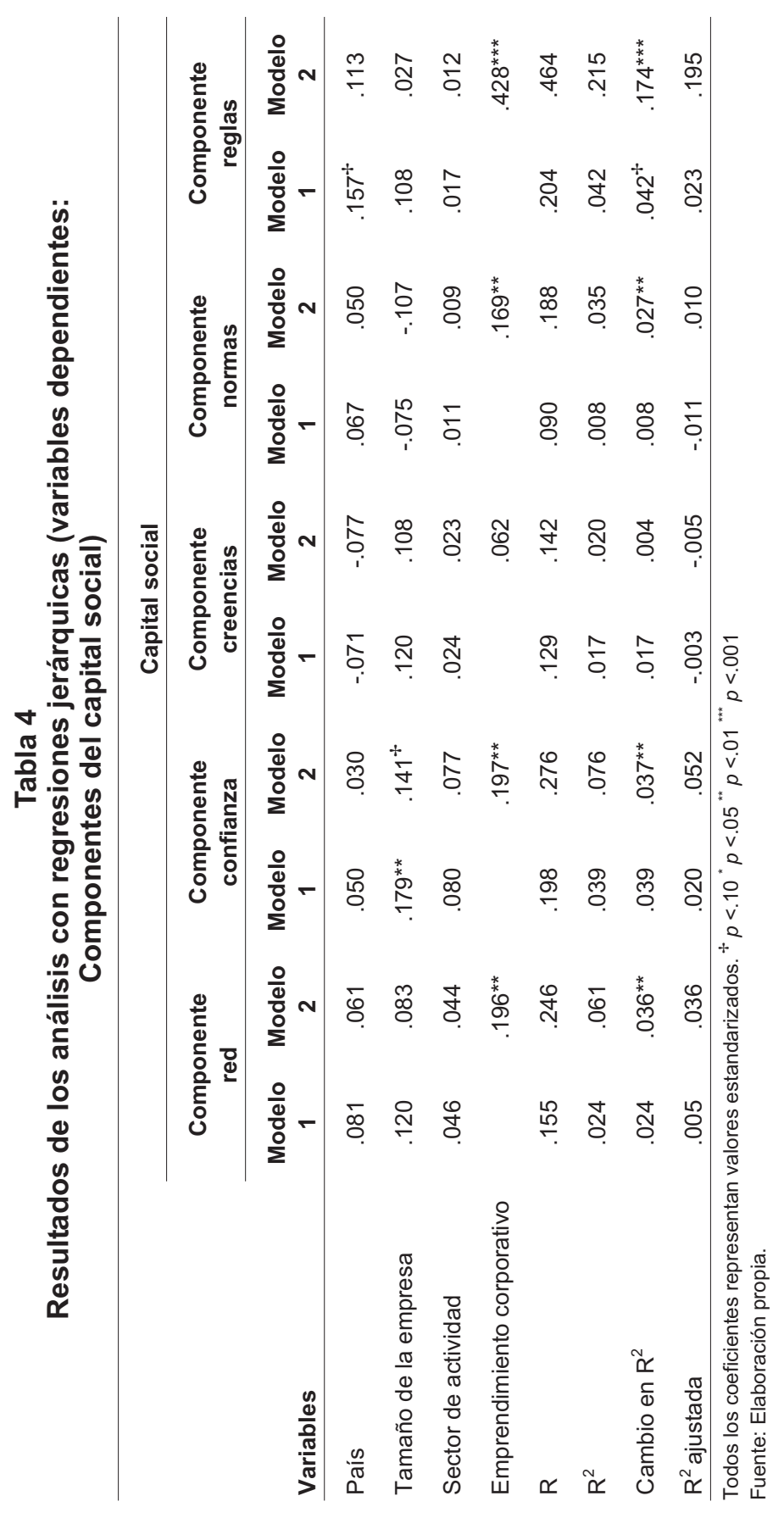


Emprendimiento corporativo y capital social: Un análisis de empresas intensivas... Delgado Márquez, Blanca L.; Delgado Márquez, Luisa y Hurtado Torres, Nuria E.

mecanismos informales que favorecen la cooperación con otras empresas. En quinto lugar, los datos sustentan la hipótesis $1 \mathrm{~d}$, esto es, la influencia positiva del emprendimiento corporativo sobre las reglas como mecanismo de control formal que favorece el intercambio de información confidencial entre empresas, evitando el riesgo de desarrollo de comportamientos oportunistas. Finalmente, la influencia del emprendimiento sobre las redes, planteada en la hipótesis 1e, queda apoyada; de este modo, las organizaciones más emprendedoras, conscientes del papel de las redes a la hora de promover acceso a información y conocimiento de utilidad y el desarrollo de nuevas oportunidades de negocio, serán más proclives a establecer y configurar más redes.

\section{Conclusiones}

El estudio del capital social ha sido objeto de un creciente interés en la literatura durante los últimos años, su desarrollo juega un papel clave como activo estratégico que permite a las empresas alcanzar ventajas competitivas. Entre los beneficios derivados del mismo se destaca cómo el capital social facilita la adquisición y explotación de conocimiento, permite el acceso a nuevos recursos financieros e influye positivamente sobre los niveles de innovación y rendimiento de las empresas.

Dada la importancia del capital social para las empresas, este trabajo avanza en el estudio de los antecedentes del mismo, analizando el papel que el emprendimiento corporativo juega sobre el capital social de las empresas y sus com- ponentes (red, confianza, creencias, normas y reglas). El análisis realizado permitió alcanzar una mejor y más holística comprensión acerca de cómo la empresa puede desarrollar una capacidad clave, como es el capital social, como medio para alcanzar ventajas competitivas en entornos altamente dinámicos y complejos.

Se concluye que el emprendimiento corporativo es clave para la acumulación y apalancamiento de recursos en las empresas, permitiéndoles desarrollar capital social, es decir, el emprendimiento corporativo ejerce una influencia positiva sobre el capital social organizativo, todo esto demostrado con evidencia empírica en el contexto de empresas intensivas en tecnología, lo que resulta especialmente relevante dado que la creación y explotación de conocimiento constituyen procesos eminentemente sociales y en los sectores intensivos en tecnología se requiere que el conocimiento se renueve de forma continua. En este sentido, una actitud más proactiva, una mayor orientación a la innovación y una mayor propensión a asumir riesgos, favorecen el desarrollo de capital social, lo que permite el acceso a nuevos conocimientos valiosos para la empresa.

Se demuestra una influencia directa, positiva y significativa del emprendimiento corporativo sobre la red de contactos que posee una empresa, la confianza sobre los miembros de la red, y las normas y las reglas que determinan las prácticas organizacionales de las empresas de la red, por el contrario, si bien estudios previos destacan el papel de las creencias como elemento guía del emprendimiento corporativo de los directivos, los 
resultados no muestran apoyo a la influencia del emprendimiento corporativo sobre las creencias compartidas. Estos resultados constituyen una aportación significativa del trabajo, puesto que se comprueba que la influencia del emprendimiento corporativo no se produce sobre todos los componentes del capital social. El hecho de que el desarrollo de esquemas compartidos de creencias entre los miembros de una organización sea un proceso dinámico, fruto de compartir sucesivas experiencias y resolver conjuntamente diversos problemas, hace que el tiempo sea una variable clave. Así pues, la no significatividad de la influencia del emprendimiento corporativo sobre el componente creencias puede estar ocasionada por el carácter transversal del análisis empírico realizado.

Adicionalmente, se derivan implicaciones prácticas tanto para los directivos de las empresas como para las instituciones que apoyan la actividad empresarial. Por un lado, se pone de relieve como el fomentar dentro de las empresas un mayor emprendimiento corporativo favorece el desarrollo de una mayor confianza entre las empresas dentro de una red de contactos, así como el establecimiento de reglas y normas comunes que faciliten la cooperación. Además, el emprendimiento corporativo permitirá el desarrollo de nuevos vínculos sociales que se traducirán en fuentes de conocimiento. Esto es especialmente importante en la situación actual donde la necesidad de adaptarse a los cambios económicos, sociales y medioambientales, pone de manifiesto que los viejos y antiguos patrones organizacionales de actuación demuestran estar quedándose obsoletos evidenciando la necesidad de un cambio. En este proceso de cambio, contar con una amplia red de vínculos sociales que constituyan diversas fuentes de conocimiento resulta de gran importancia. Por otro lado, en el actual contexto socio-económico, el impulso de las instituciones en favor de un mayor emprendimiento corporativo por parte de las empresas, resulta clave para poder afrontar las nuevas necesidades de los mercados.

A pesar de lo comentado con anterioridad, dado que la muestra empleada en este trabajo se centra a empresas de alta tecnología, los resultados pueden no ser directamente extrapolables a otras empresas de sectores no intensivos en tecnología. Futuras investigaciones tratarán de abordar este aspecto ampliando el ámbito de estudio a otros sectores. Es de señalar que para medir los conceptos, emprendimiento corporativo y capital social, se ha utilizado como única fuente de información al director general de las empresas de la muestra. Trabajos posteriores pueden recabar información de índole secundaria que complemente la información obtenida en la encuesta. Por último, cabe mencionar que entre las propuestas o futuras líneas de investigación que se pueden derivar de este trabajo, consideramos especialmente interesante analizar el potencial efecto moderador de la cultura del país origen de la empresa en la relación entre emprendimiento corporativo y capital social. 
Emprendimiento corporativo y capital social: Un análisis de empresas intensivas...

Delgado Márquez, Blanca L.; Delgado Márquez, Luisa y Hurtado Torres, Nuria E.

\section{Referencias bibliográficas}

Álvarez, Sharon A. y Busenitz Lowell W. (2001). The entrepreneurship of resource-based theory. Journal of Management, Vol. 27, Sage Publications, pp. 755-775.

Aldrich, Howard y Martínez, Martha (2003). Entrepreneurship as social construction. The international handbook of entrepreneurship, Z. J. Acs and D.B. Audretsch, Dordrecht: Kluwer Academic Publishers.

Anandarajan, Asokan; Chiang, Shuling y Lee, Picheng (2010). R\&D tax credit and operating performance: Implications for managers. Management Decision, Vol. 48, No. 8, Emerald Group Publishing Limited, pp. 1198-1211.

Armstrong, Joshua Scott y Overton, Terry (1977). Estimating nonrespone bias in mail surveys. Journal of Marketing Research, Vol. 14, No. 3, USA, American Marketing Association, pp. 396-402.

Audretsch, David B. y Aldridge, Taylor (2012). Transnational social capital and scientist entrepreneurship. Journal of Management and Governance, Vol. 16, Holanda, Springer Science and Media Business, pp. 369-376.

Baer, Markus y Frese, Michael (2003). Innovation is not enough: Climate for iniciative and psychological safety, process innovations, and firm performance. Journal of Organizational Behavior, Vol. 24, USA, John Wiley and Sons, Inc., pp. 45-68.

Bourdieu, Pierre (1986). The forms of capital. Handbook of theory and research for the sociology of education, New York, Editorial Greenwood Press, pp. 241-258.

Burt, Ronald Stuart (1992). Structural holes: The social structure of competition, United Kingdom, Harvard University Press.
Burt, Ronald Stuart (1997). The contingent value of social capital. Administrative Science Quarterly, Vol. 42, No. 2, USA, Administrative Science Quarterly, pp. 339-365.

Burt, Ronald Stuart (2001). Structural holes versus network closure as social capital. Social capital: Theory and research, USA, Transaction $\mathrm{Pu}$ blishers.

Carpenter, Mason; Li, Mingchiang y Jiang, Han (2012). Social network in organizational contexts: A systematic review of methodological issues and choices. Journal of Management, Vol. 38, No. 4, Southern Management Association, pp. 1328-1360.

Cegarra Navarro, Juan Gabriel; Sanchez Vidal, M. Eugenia y Cegarra Leiva, David (2011). Balancing exploration and exploitation of knowledge through an unlearning context: An empirical investigation in SMEs. Management Decision, Vol. 49, No.7, Emerald Group Publishing Limited, pp. 10991119.

Cohen, Jacob y Cohen, Patricia (1983). Applied multiple regression/correlation analysis for the behavioural science, Hillsdale, N.J. Erlbaum.

Coleman, James Samuel (1988). Social capital in the creation of human capital. The American Journal of Sociology, Vol. 94, No. 1, USA, The University of Chicago Press, pp. 95-120.

Coleman, James Samuel (1990). Foundations of social theory, United Kingdom, Harvard University Press.

Covin, Jeffrey y Miles, Morgan (1999). Corporate entrepreneurship and the pursuit of competitive advantage. Entrepreneurship Theory and Practice, Vol. 23, No. 1, United Kingdom, WileyBlackwell, pp. 47-63.

Covin, Jeffrey y Slevin, Dennis (1988). The influence of organization structure on the 
utility of an entrepreneurial top management style. Journal of Management Studies, Vol. 25, No. 3, United Kingdom, Wiley-Blackwell, pp. 251-334.

Covin, Jeffrey y Slevin, Dennis (1989). Strategic management of small firms in hostile and benign environments. Strategic Management Journal, Vol. 10, No. 1, USA, John Wiley and Sons, pp. 75-87.

Crant, J. Michael (2000). Proactive behavior in organizations. Journal of Management, Vol. 26, No. 3, Sage Publications, pp. 435-462.

Dakhli Mourad y de Clercq Dirk (2004). Human capital, social capital, and innovation: A multi-country study. Entrepreneurship and Regional Development, Vol. 16, No. 2, United Kingdom, Routledge, pp. 107-128.

Delgado Márquez, Blanca Luisa; Hurtado Torres, Nuria Esther y Aragón Correa, Juan Alberto (2012). The dynamic nature of trust transfer: Measurement and the influence of reciprocity. Decision Support Systems, doi:10.1016/j.dss.2012.05.008.

Dess, Gregory; Lumpkin, Taylor y Covin, Jeffrey (1997). Entrepreneurial strategy making and firm performance: Tests of contingency and configurational models. Strategic Management Journal, Vol. 18, No. 9, USA, John Wiley and Sons, pp. 677-695.

Doh, Soogwan y Zolnik, Edmund (2011). Social capital and entrepreneurship: An exploratory analysis. African Journal of Business Management, Vol. 5, No. 12, pp. 4961-4975.

Fernández, Gladys; Narváez, Mercy y Senior, Alexa (2010). Organizaciones cooperativas en el contexto del desarrollo local: Una aproximación para su estudio. Revista Venezolana de Gerencia, Vol. 15, No. 49, Venezuela, Centro de Estudios de la Empresa, Universidad del Zulia, pp. 87-102.
Frohlich, Markham y Westbrook, Roy (2002). Demand chain management in manufacturing and services: Web-based integration, drivers and performance. Journal of Operations Management, Vol. 20, No. 6, USA, Elsevier, pp. 729-745.

Fukuyama, Francis (1995). Trust: The social virtues and the creation of prosperity, United Kingdom, London: Hamish Hamilton.

Fukuyama, Francis (1997). Social capital and the modern capitalist economy: Creating a high trust workplace. Stern Business Magazine, Vol. 4, No. 1, USA, Stern School of Business, New York University.

Fukuyama, Francis (1999). The great disruption: Human nature and the reconstitution of social order, New York: Free Press.

Fukuyama, Francis (2001). Social capital, civil society and development. Third World Quarterly, Vol. 22, No. 1, United Kingdom, Routledge, pp. 7-20.

Gamboa Cáceres, Teresa; Arellano Rodríguez, Madelein y Nava Vázquez, Yuneska (2001). Estrategias empresariales: Aproximación a una tipología. Revista Venezolana de Gerencia, Vol. 5, No. 2, Venezuela, Centro de Estudios de la Empresa, Universidad del Zulia, pp. 132-154.

Govea, Héctor y Rodríguez, Isabel (2009). Capital social, desarrollo endógeno y gestión de demandas ciudadanas. Revista

Venezolana de Gerencia, Vol. 14, No. 45, Centro de Estudios de la Empresa, Universidad del Zulia, pp. 55-77.

Gulati, Ranjay (1995). Social structure and alliance formation patterns: A longitudinal analysis. Administrative Science Quarterly, Vol. 40, No. 4, Sage Publications, pp. 619-652.

Hair, Joseph; Black, Bill; Babin, Barry; Anderson, Rolph y Tatham, Ronald (2006). 
Emprendimiento corporativo y capital social: Un análisis de empresas intensivas... Delgado Márquez, Blanca L.; Delgado Márquez, Luisa y Hurtado Torres, Nuria E.

Multivariate data analysis, Upper Saddle River, Prentice Hall.

Hayton, James y Kelley, Donna (2006). A competency based framework for promoting corporate entrepreneurship. Human Resource Management, Vol. 45, No. 3, USA, John Wiley and Sons, pp. 407-427.

Hoang, Ha y Antonic, Bostjan (2003). Network-based research in entrepreneurship: A critical review. Journal of Business Venturing, Vol. 18, No. 2, Holanda, Elsevier, pp. 165-187.

Hotho, Sabine y Champion, Katherine (2011). Small businesses in the new creative industries: Innovation as a people management challenge. Management Decision, Vol. 49, No. 1, Emerald Group Publishing Limited, pp. 29-54.

Kale, Prashant; Singh, Harbir y Perlmutter, Howard (2000). Learning and protection of proprietary assets in strategic alliances: Building relational capital. Strategic Management Journal, Vol. 21, No. 3, USA, John Wiley and Sons, pp. 217-237.

Kogut Bruce y Zander Udo (1992). Knowledge of the firm, combinative capabilities, and the replication of technology. Organization Science, Vol. 3, No. 3, The Institute of Management Sciences, pp. 383-397.

Konrad, Alison y Fran Linnehan (1995). Formalized HRM structures: Coordinating equal employment opportunity or concealing organizational practice? Academy of Management Journal, Vol. 38, United States of America, Academy of Management, pp. 787-820.

Knight, Gary (1997). Cross-cultural reliability and validity of a scale to measure firm entrepreneurial orientation. Journal of Business Venturing, Vol. 12, No. 3, Holanda, Elsevier, pp. 213-225.
Kuratko, Donald; Ireland, Duane; Covin Jeffrey y Hornsby, Jeffrey (2005). A model of middle-level managers' entrepreneurial behavior. Entrepreneurship Theory and Practice, Vol. 26, No. 6, United Kingdom, Wiley-Blackwell, pp. 699-716.

Kymberly, John y Evanisko, Michael (1981). Organizational innovation: The influence of individual, organizational, and contextual factors on hospital adoption of technological and administrative innovations. Academy of Management Journal, Vol. 24, No. 4, USA, Academy of Management, pp. 689-713.

Lane, Peter y Lubatkin, Michael (1998). Relative absorptive capacity and interorganizational learning. Strategic Management Journal, Vol. 19, No. 5, USA, John Wiley \& Sons, pp. 461-477.

$\mathrm{Li}$, Suhong y Lin, Binshan (2006). Accessing information sharing and information quality in supply chain management. Decision Support Systems, Vol. 42, Holanda, Elsevier, pp. 1641-1656.

Lumpkin, G. Tom y Dess, Gregory (1996). Clarifying the entrepreneurial orientation construct and linking it to performance. Academy of Management Review, Vol. 21, No. 1, Academy of Management, pp. 135-172.

Luque-Martínez, Teodoro (2000). Técnicas de análisis de datos en investigación de mercados, Madrid, Pirámide.

Mainardes, Emerson Wagner; Alves, Helena y Raposo, Mario (2011). Stakeholder theory: Issues to resolve. Management Decision, Vol. 49, No. 2, Emerald Group Publishing Limited, pp. 226-252.

McElroy, Mark; Jorna, René y van Engelen, Jo (2006). Rethinking social capital theory: A knowledge management perspective. Journal of Knowledge Management, Vol. 10, No. 5, Kempston, United Kingdom, pp. 124-136. 
Miller, Danny (1983). The correlates of entrepreneurship in the three types of firms. Management Science, Vol. 29, INFORMS, pp. 770-791.

Morris, Henry, Kuratko, Donald y Covin, Jeffrey (2008). Corporate entrepreneurship and innovation: Entrepreneurial development within organizations, Mason, Ohio, Thomson/ South-Western.

Nahapiet, Janine y Ghoshal, Sumantra (1998). Social capital, intellectual capital, and the organizational advantage. Academy of Management Review, Vol. 23, No. 2, USA, Academy of Management, pp. 242-266.

Narayan, Deepa (1999). Bonds and bridges: Social capital and poverty, Washington DC: The World Bank, Policy Research Working Paper, No. 2167.

Ostrom, Elinor (2005). Understanding institutional diversity, Princeton, USA, Princeton University Press.

Payne, Tyge; Moore, Curt; Griffis, Stanley y Autry, Chad (2011). Multilevel challenges and opportunities in social capital research. Journal of Management, Vol. 37, No. 2, United Kingdom, Sage Publications, pp. 491-520.

Peng, Mike W. y Luo, Yadong (2000). Managerial ties and firm performance in a transition economy: The nature of a micromacro link. Academy of Management Journal, Vol. 43, No. 3, United States of America, Academy of Management, pp. 486-501.

Phan, Phillip; Wright, Mik; Ucbasaran, Deniz y Tan, Wee-Liang (2009). Corporate entrepreneurship: Current research and future directions. Journal of Business Venturing, Vol. 24, No. 3, Holanda, Elsevier, pp. 197-205.

Podolny, Joel M. (2001). Networks as pipes and prisms of the market. The American Journal Pychology, No. 107,
United States of America, University of Illinois Press, pp. 33-60.

Portes, Alejandro (1998). Social capital: Its origins and applications in modern sociology. Annual Review of Sociology, Vol. 24, No. 1, USA, Annual Reviews Inc., pp. 1-24.

Putnam, Robert (1993). The prosperous community: social capital and public life. The American Prospect, Vol. 13, No. 4, USA. The American prospect, Inc., pp. 35-42.

Putnam, Robert (1995). Tuning in, tuning out: The strange disappearance of social capital in America. Political Science and Politics. Vol. 28, No. 4, USA, American Political Science Association, pp. 664-683.

Rauch, Andreas; Wiklund, Johan; Lumpkin G. Tom y Frese, Michael (2009). Entrepreneurial orientation and business performance: An assessment of past research and suggestions for the future. Entrepreneurship Theory and Practice, Vol. 33, No. 3, Baylor University, pp. 761-787.

Rodríguez Ramírez, Alfonso; Rodríguez Orejuela, Augusto y Guillermo Murillo Vargas (2010). New perspectives for the managerial entrepreneurship. International Entrepreneurship and Management Journal, Vol. 6, No. 2, Holanda, Springer Science and Business Media, pp. 203-219.

Romero, Gerardo (2006). Principios y valores éticos en las organizaciones bancarias respecto a la calidad del servicio y atención al cliente. Revista Venezolana de Gerencia, Vol. 11, No. 36, Venezuela, Centro de Estudios de la Empresa, Universidad del Zulia, pp. 638-651.

Rousseau, Denise; Sitkin, Sim; Burt, Ronald Stuart y Camerer Colin (1998). Not so different after all: A crossdiscipline view of trust. Academy of Manage- 
Emprendimiento corporativo y capital social: Un análisis de empresas intensivas... Delgado Márquez, Blanca L.; Delgado Márquez, Luisa y Hurtado Torres, Nuria E.

ment Review, Vol. 23, No. 3, United States of America, Academy of Management, pp. 393-404.

Sánchez de Pablo González del Campo, Jesús David; Škerlavaj, Miha; Guadamillas Gómez, Fátima y Dimovski, Vlado (2010). Redes de aprendizaje intraorganizativo: Análisis exploratorio de un caso. Revista Venezolana de Gerencia, Vol. 15, No. 49, Venezuela, Centro de Estudios de la Empresa,Universidad del Zulia, pp. 11-29.

Saxenian, Annalee (1994). Regional advantage: Culture and competition in Silicon Valley and Route 128, Cambridge, MA: Harvard University Press.

Schein, Edgar (1985). Organizational culture and leadership. San Francisco: Jossey-Bass Publishers.

Shane, Scott y Cable, Daniel (2002). Network ties, reputation, and the financing of new ventures. Management Science, Vol. 48, No.3, United States of America, INFORMS, pp. 364-381.

Shane, Scott y Venkataraman, Sankaran (2000). The promise of entrepreneurship as a field of research. Academy of Management Review, Vol. 25 , No. 1, Academy of Management, pp. 217-226.

Shipilov, Andrew V. (2009). Firm scope experience, historic multimarket contact with partners, centrality, and the relationship between structural holes and performance. Organization Science, Vol. 20, No. 1, United States of America, INFORMS, pp. 85-106.

Simsek, Zeky y Ciarian, Heavy (2011). The mediating role of knowledge-based capital for corporate entrepreneurship effects on performance: A study of small-to medium-sized firms. Strategic Entrepreneurship Journal, Vol. 5, No. 1, USA, Strategic Management Society, pp. 81-100.
Smedlund, Anssi (2008). The knowledge system of a firm: Social capital for explicit, tacit and potential knowledge. Journal of Knowledge Management, Vol. 12, No. 1, Kempston, United Kingdom, pp. 63-77.

Teng, Bing-Sheng (2007). Corporate entrepreneurship activities through strategic alliances: A resource-based approach toward competitive advantage. Journal of Management Studies, Vol. 44, No. 1, United Kingdom, WileyBlackwell, pp. 119-142.

Thorgren, Sara; Örtqvist, Daniel y Wincent, Joakim (2009). A cause-effect of inter-firm networking and corporate entrepreneurship: Initial evidence of self-enforcing spirals. Journal of Developmental Entrepreneurship, Vol. 14, No. 4, USA, World Scientific Publishing Company, pp. 355-373.

Westhpal, James y Fredrickson, James (2001). Who directs strategic change? Director experience, the selection of new CEOs, and change in corporate strategy. Strategic Management Journal, Vol. 22, No. 12, USA, John Wiley and Sons, pp. 1113-1137.

Williamson, Oliver (1993). Calculativeness, trust, and economic organization. Journal of Law and Economics, Vol. 34, United States of America, University of Chicago Law School, pp. 453-502.

Woolcock, Michael (1998). Social capital and economic development: Toward a theoretical synthesis and policy framework. Theory and Society, Vol. 27, No. 2, Alemania, Springer, pp. 151-208.

Woolcock, Michael y Narayan, Deepa (2000). Social capital: Implications for development theory, research, and policy. The World Bank Research Observer, Vol. 15, No. 2, pp. 225-249.

Yli-Renko, Helena; Autio, Erkko y Sapienza, Harry (2001). Social capital, knowl- 
edge acquisition and knowledge explotation in young technology-based firms. Strategic Management Journal, Vol. 22, No. 6/7, United States of America, John Wiley and Sons, pp. 587-613.
Zahra, Shaker; Nielsen, Anders y Bogner, William (1999). Corporate entrepreneurship, knowledge and competence development. Entrepreneurship theory and practice, Vol. 23, No. 4, United Kingdom, Wiley-Blackwell, pp. 169-189. 